\title{
Förderpreis Nephrologische Pflege 2014
}

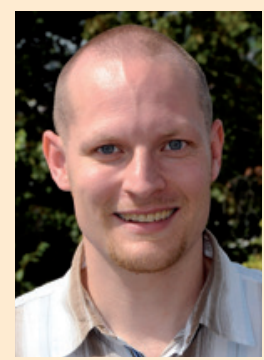

Christian Schäfer, Stuttgart
Im Rahmen der 2014 erstmals - und sehr erfolgreich - durchgeführten AfnP Dialyse Basic Days in Fulda konnte ich zusammen mit Marion Bundschu, 1. Vorsitzende der AfnP, den Förderpreis Nephrologische Pflege 2014 vergeben. Das Preisgeld von 1500 Euro ging verdient an die Autorin der in der Bewertung erstplatzierten Facharbeit zum Thema „Natriumprofile - Ausschöpfung technischer Möglichkeiten moderner Dialysegeräte“. Auch die Arbeiten auf den Plätzen 2 („Vermeidung der Komplikation intradialytische Hypotonie“) und 3 („Dialyse im Wandel - Neuer Anspruch für Pflegende“) waren von hoher Qualität, was wir mit Thieme Büchergutscheinen honorierten. Einen kurzen Überblick über die Beiträge auf den ersten 3 Plätzen und deren Autoren sowie eine Ankündigung zum diesjährigen Förderpreis Nephrologische Pflege finden Sie auf den Seiten 8 und 10 sowie auf Seite 15 in dieser Ausgabe. Ab Seite 24 können Sie schließlich die Artikel lesen, die auf den 3 „Siegertreppchen-Beiträgen“ basieren.

Ich freue mich sehr, dass wir als Verlag in unserer Funktion als Sponsor für diesen Preis und mit der Veröffentlichung der besten Artikel nun schon zum neunten Mal dazu beitragen können, dass pflegerisches Fachwissen wissenschaftlich aufbereitet bei der Zielgruppe - Pflegekräften in der Nephrologie - ankommt. Dies hilft dabei, die schon lange unbefriedigend niedrige Zahl an pflegewissenschaftlichen Veröffentlichungen v.a. im deutschsprachigen Raum zu erhöhen und die Qualität der Pflege zu verbessern. Vielleicht haben auch Sie Lust, einen Beitrag für die Bewerbung um den 2015er-Preis einzureichen? Bis zum 01.08. haben Sie noch Zeit, ihn an die Geschäftsstelle der AfnP zu senden. Die Kriterien sowie die notwendigen Adressen und weitere Informationen finden Sie unter www.afnp.de/pflegepreis.php.

Wir haben in den letzten Jahren einiges dafür getan, die Dialyse aktuell für nephrologische Pflegekräfte noch mehr als zuvor zu einer Quelle wichtiger Informationen und nützlichem Wissen für Theorie und Praxis zu machen. So finden Sie in der Rubrik ,Journal-Club Pflege“ in etwa jeder zweiten Ausgabe pflegewissenschaftliche Studien aus internationalen Publikationen kurz und übersichtlich aufbereitet (in dieser Ausgabe auf Seite 23). Außerdem konnten wir mit der kräftigen Hilfe engagierter Autoren die Zahl wissenschaftlicher Beiträge mit pflegerischem Bezug in der Rubrik „Pflegeserie“ erhöhen. Auch 2015 werden Sie hierzu wieder etwas in der Dialyse aktuell finden können. Schon seit Jahren bekommen Pflegekräfte für die Mitgliedschaft in der AfnP oder dem fnb (und somit dem Bezug der Dialyse aktuell) oder über ein Abonnement der Zeitschrift jährlich 3 Punkte für die freiwillige Registrierung beruflich Pflegender. Und natürlich finden Sie in jeder Ausgabe Neuigkeiten aus den Fachgesellschaften inkl. Kongressankündigungen und -nachberichten.

Eine neue Rubrik kann ich Ihnen wärmstens ans Herz legen: den „Expertentipp“, der Ihnen in regelmäßigen Abständen Aspekte verschiedener, sich abwechselnder Themen kurz und übersichtlich mit Praxisbezug näherbringt. Das Thema „Nierentransplantation und Immunsuppression“ macht in dem vorliegenden Heft auf Seite 20 den Anfang. Einen weiteren sehr interessanten Beitrag finden Sie gleich auf den Folgeseiten, und zwar in der Rubrik „Magazin“ zur „Zukunft der Dialyse“. Ich wünsche Ihnen eine angenehme Lektüre der ersten Ausgabe der Dialyse aktuell des Jahrgangs 2015! 